\title{
Social deprivation increases cardiac hospitalisations in chronic heart failure independent of disease severity and diuretic non-adherence
}

\author{
A D Struthers, G Anderson, P T Donnan, T MacDonald
}

\begin{abstract}
Objective-To examine whether social deprivation has any independent effect on emergency cardiac hospitalisations in patients with chronic heart failure $(\mathrm{CHF})$.

Design-Cohort study of 478 patients with CHF who had been hospitalised before 1993 and who were followed up during 1993 and 1994.

Setting-Emergency admissions within Tayside acute hospitals.

Patients-478 CHF patients who had a previous myocardial infarction, a previous CHF admission, and were on diuretic treatment.

Main outcome measures-Emergency hospital admissions are divided into those for all causes and those for cardiac causes only.

Results-Social deprivation was significantly associated with an increase in the number of cardiac hospitalisations $(\mathrm{p}=0.007)$. This effect was mainly caused by increasing the proportion of patients hospitalised in each deprivation category (26\% in deprivation category $1-2$ versus $40 \%$ in deprivation category $5-6, \mathrm{p}=0.03$ ). This effect of deprivation was independent of disease severity, as judged by the dose of prescribed diuretic, the death rate, and the duration of each hospital stay. Non-adherence with diuretic treatment could not account for these findings either. Conclusions-Social deprivation increases the chance of a CHF patient being rehospitalised independently of disease severity. Possible explanations are that doctors who look after socially deprived patients have a lower threshold for cardiac hospitalisation of their patients, or that social deprivation alters the way a CHF patient accesses medical care during decompensation. Understanding how social deprivation influences both doctor and patient behaviour in the prehospital phase is now crucial in order to reduce the amplifying effect that social deprivation appears to have on cardiac hospitalisations.

(Heart 2000;83:12-16)

Keywords: chronic heart failure; hospitalisations; social deprivation
\end{abstract}

Department of Clinical Pharmacology and Therapeutics, Ninewells Hospital, Dundee DD1 9SY, UK A D Struthers

Medicines Monitoring Unit, Ninewells Hospital

G Anderson

P T Donnan

T MacDonald

Correspondence to: Professor Struthers

Accepted for publication 16 August 1999
It is now well established that social deprivation is associated with an increased burden of disease in the population, especially coronary disease. ${ }^{1}$ Social deprivation is also associated with an increase in acute emergency hospital admissions. ${ }^{2-4}$ However, use of health services does not always parallel clinical need. Indeed, a great unknown is whether the number of acute hospitalisations in the socially deprived is appropriate for their increased disease burden or whether it is lower or higher than would be expected. In other words, are "demand" and "need" matched?

Clearly the answer to this question may vary from one disease to another; we therefore chose to study this question for chronic heart failure $(\mathrm{CHF}) . \mathrm{CHF}$ is a particularly appropriate disease to study as it accounts for a sizeable proportion of all acute medical emergencies, it is costly to the National Health Service ( $£ 360$ million/year in 1993), and the majority of costs for $\mathrm{CHF}$ comes from repeated emergency hospitalisations. ${ }^{5}$ Its other attraction for this study is that disease severity for CHF can be estimated fairly objectively in large databases by the dose of diuretic prescribed and by death rates.

Our study design enabled us to answer another key question in heart failure. Since half of the excess coronary mortality in the socially deprived is attributable to known uncorrected risk factors such as smoking, it has been assumed that the socially deprived are less health conscious. If so, one might expect that adherence to prescribed medication would be less in the socially deprived. We therefore set out to examine whether non-adherence with treatment was indeed greater in the socially deprived than in other socioeconomic groups.

\section{Methods}

Our study was carried out using the record linkage system of the medicines monitoring unit, University of Dundee. The collection methods for this database have been described in further detail elsewhere. ${ }^{6}$

INCLUSION CRITERIA

Patients included in the study had to fulfil the following criteria: (1) they were admitted to a Tayside hospital with a myocardial infarction (International Classification of Diseases, ninth revision (ICD-9) code 410.9) between 1 January 1989 and 31 December 1992; (2) they were subsequently admitted to a Tayside hospital for chronic heart failure (ICD-9 code 428.0, 428.1, 429.3) between 1 January 1989 and 31 December 1992; (3) in order for the diuretic adherence data to cover a reasonable period of time, three or more prescriptions had to have been dispensed to patients in Tayside between January 1993 and January 1994.

HOSPITALISATION DATA

We recorded information for all emergency admissions, for cardiac emergency admissions, and for deaths from 1 January 1993 to 31 
December 1994, inclusive. The study was performed over a specified period so that diuretic adherence could be calculated for a time period which corresponded with the hospitalisation data. Emergency admissions were recorded for all causes and for cardiac causes as described below.

ASSIGNING DEPRIVATION CATEGORIES

A Carstairs score was assigned to each person via postcodes using the record linkage database. Deprivation scores from 1-7 are assigned to postcode sectors as described by McLoone. ${ }^{7}$ Areas defined as deprivation category 1 are the most affluent areas, and those defined as deprivation category 7 are the most deprived areas.

CALCULATING DIURETIC ADHERENCE OVER THE STUDY PERIOD

Our general methodology for measuring adherence to treatment has been described before. ${ }^{7}$ The number of days when medication was available to each individual was calculated and became the numerator for diuretic adherence. The length of time over which each patient's adherence was assessed was determined by subtracting the date that the first prescription was issued in the study period from the date of the last prescription in the study period or the date of death, whichever is the earlier; hence the denominator takes into account death before the end of the study. To prevent an underestimate of drug adherence, the total number of days in hospital during the study period was subtracted from the denominator. Diuretic adherence was then expressed as the percentage of time within the study when the individual had diuretic drugs available at their home.

STATISTICAL METHODS

Hospitalisation data were obtained from the standardised morbidity record 1 (SMR1) which gives the date of admission, the type of admission (emergency or not), and the primary reason for admission according to the ICD-9 code. The ICD-9 codes used to define a cardiac admission were 410, 411, 413, 414.0, $414.1,414.8,414.9,425,427,428.0,428.1$, $428.9,429.1,429.2,429.3,429.4,429.5$, $429.6,429.8$, and 429.9. Each record of the SMR1 is based on a finished consultant episode and so is not the same as the one continuous stay in hospital. If a patient is transferred to another consultant within the same period of stay in hospital this is counted as another episode.

Diuretic dose - a measure of severity - was obtained and categorised as $\leqslant 39 \mathrm{mg}$ frusemide equivalent, $40-79 \mathrm{mg}, 80-119 \mathrm{mg}, 120$ $159 \mathrm{mg}$, or $\geqslant 160 \mathrm{mg}$. Adherence with diuretic treatment was defined as above and for the purposes of presentation was split into the categories $\leqslant 79 \%, 80-124 \%$, and $>124 \%$. The number and proportion of subjects who had the two outcomes were tabulated by diuretic category and compliance category. The number and proportion of subjects who had an emergency cardiac admission or any emergency admission were tabulated by deprivation category. Deprivation was measured by the
Carstairs deprivation code which has values from 1 (affluent) to 7 (most deprived). ${ }^{7}$ In this study there were no subjects in deprivation category 7 . For the purposes of tabulation categories 1 and 2,3 and 4 , and 5 and 6 were combined into three categories, while for regression analysis all deprivation categories were used to increase power. Tests for trends in proportions were carried out using the Cochran-Armitage test. In addition, the number of admissions per person were calculated and regressed on deprivation score to test for trends across deprivation categories. A logarithmic transformation of the number of admissions was used in this regression analysis.

Cox's proportional hazards model was used to assess trends in deprivation in relation to the time to the first emergency admission (cardiac and any admission). In this way those who died over the two year follow up would be censored at time of death. These analyses were adjusted for age, sex, diuretic category, and compliance category. Results are expressed as risk ratios with $95 \%$ confidence intervals (CI). All analyses were carried out using SAS, version 6.12.

\section{Results}

The cohort comprised $478 \mathrm{CHF}$ patients $(52 \%$ male); ages ranged from 46-90 years with $80 \%$ $>65$ years old. Patients were distributed across deprivation categories $1-6$ but no patient was in category 7 . However, a deprivation category could not be assigned in 16 patients.

Tables 1 and 2 show that deprivation was significantly associated with an increased likelihood of a patient having a cardiac hospitalisation, but it had no significant effect on the likelihood of a patient being hospitalised for any cause. This was despite there being no association between deprivation and the death rate, the prescribed dose of diuretics, the adherence of the patient to that dose of diuretic, or to the duration of hospital stay (table 1). Therefore, social deprivation was specifically associated with cardiac hospitalisations and this is unlikely to be attributable to disease severity as judged by death rates, the dose of prescribed diuretic, or the length of stay in hospital.

After we found that social deprivation was associated with a significantly greater absolute number of cardiac hospitalisations, we then examined whether the increased number of hospitalisations in the socially deprived was caused by a higher proportion of those patients being admitted or whether it was caused by more frequent hospitalisations in the same proportion of patients. Table 1 shows that both are associated with an increase although it is only in the former that the increase is significant. Interestingly, the opposite pattern was seen for total emergency admissions - that is, as far as emergency admissions for all causes is concerned, social deprivation was associated with a significant increase in repeated admissions rather than a higher proportion of patients being admitted. A Cox's proportional hazards model was also performed in order to check that our findings were not influenced by any differential time to death in each depriva- 
Table 1 Association between social deprivation, cardiac emergency hospitalisations, severity of disease, and diuretic adherence

\begin{tabular}{|c|c|c|c|c|}
\hline & \multicolumn{3}{|c|}{ Deprivation category } & \multirow[b]{2}{*}{$p$ Value } \\
\hline & $\begin{array}{l}1-2 \\
(n=106)\end{array}$ & $\begin{array}{l}3-4 \\
(n=204)\end{array}$ & $\begin{array}{l}5-6 \\
(n=152)\end{array}$ & \\
\hline \multicolumn{5}{|l|}{ Cardiac emergency hospitalisation data } \\
\hline Number of cardiac hospitalisations per patient (geometric mean) in each group & 0.71 & 0.81 & $0.91^{\star}$ & 0.007 \\
\hline Patients with any cardiac hospitalisation $(\%)$ & 26 & 34 & $40^{\star}$ & 0.03 \\
\hline Number of cardiac hospitalisations per hospitalised patient (geometric mean) & 1.38 & 1.50 & 1.66 & NS \\
\hline \multicolumn{5}{|l|}{ Measures of severity } \\
\hline Percentage died $(\%)$ & 16 & 20 & 18 & NS \\
\hline Average (SD) diuretic dose frusemide equivalents (mg) & $85(38)$ & $78(46)$ & $83(39)$ & NS \\
\hline Median length of hospital stay (days) & 6 & 6.5 & 6 & NS \\
\hline \multicolumn{5}{|l|}{ Adherence measure } \\
\hline Mean (SD) diuretic adherence (\%) & $96(29)$ & $93(26)$ & $99(36)$ & NS \\
\hline
\end{tabular}

${ }^{\star}$ Cochran-Armitage test for trend.

Table 2 Association between social deprivation, all emergency hospitalisations, severity of disease, and diuretic adherence

\begin{tabular}{|c|c|c|c|c|}
\hline & \multicolumn{3}{|c|}{ Deprivation category } & \multirow[b]{2}{*}{ p Value } \\
\hline & $\begin{array}{l}1-2 \\
(n=106)\end{array}$ & $\begin{array}{l}3-4 \\
(n=204)\end{array}$ & $\begin{array}{l}5-6 \\
(n=152)\end{array}$ & \\
\hline \multicolumn{5}{|l|}{ All emergency hospitalisation data } \\
\hline Number of total hospitalisations per patient (geometric mean) in each group & 1.21 & 1.25 & 1.41 & NS \\
\hline Patients hospitalised (\%) & 56 & 57 & 58 & NS \\
\hline Number of hospitalisations per hospitalised patient (geometric mean) & 1.86 & 1.91 & $2.38^{\star}$ & 0.03 \\
\hline \multicolumn{5}{|l|}{ Measures of severity } \\
\hline Percentage died $(\%)$ & 16 & 20 & 18 & NS \\
\hline Average (SD) diuretic dose frusemide equivalents (mg) & $85(38)$ & $78(46)$ & $83(39)$ & NS \\
\hline Median length of hospital stay (days) & 7 & 7 & 7 & NS \\
\hline \multicolumn{5}{|l|}{ Adherence measure } \\
\hline Mean (SD) diuretic adherence (\%) & $96(29)$ & $93(26)$ & $99(36)$ & NS \\
\hline
\end{tabular}

^Cochran-Armitage test for trend.

tion category and to adjust for age and sex. Table 3 shows that in this particular model of time to first hospitalisation, social deprivation was the only significant determinant, after adjustment for all factors. The risk ratio for cardiac hospitalisations for an increase of one category of social deprivation was $1.11(95 \%$ CI 1.002 to 1.224 ).

Table 4 shows that it is only when the frusemide dose was $>160 \mathrm{mg} /$ day that a significant increase occurred in either cardiac hospitalisations or in hospitalisations for any cause. This expected finding is reassuring and gives added confidence in the data. The impact on cardiac

Table 3 Cox's proportional hazard model for time to first emergency hospitalisation across all six deprivation categories

\begin{tabular}{lll}
\hline & $\begin{array}{l}\text { Cardiac emergency admissions } \\
\text { relative risk (95\% CI) }\end{array}$ & $\begin{array}{l}\text { Total emergency admissions } \\
\text { relative risk (95\% CI) }\end{array}$ \\
\hline Unadjusted & $1.11(1.004$ to 1.225$)$ & $1.007(0.933$ to 1.008$)$ \\
Adjusted for age, sex & $\mathrm{p}=0.041$ & $\mathrm{p}=0.851$ \\
$1.11(1.002$ to 1.224$)$ & $\mathrm{p}=0.740$ \\
\hline
\end{tabular}

Deprivation was the only significant factor in the Cox's model. Neither age, sex, diuretic adherence nor diuretic dose were significant in this time to first hospitalisation analysis.

Table 4 Effect of diuretic dose and diuretic adherence on cardiac hospitalisations

\begin{tabular}{lll}
\hline $\begin{array}{l}\text { Diuretic dose (frusemide } \\
\text { equivalents mg/day) }\end{array}$ & $\begin{array}{l}\text { Patients undergoing an emergency } \\
\text { cardiac hospitalisation (\%) }\end{array}$ & $\begin{array}{l}\text { Patients undergoing any } \\
\text { emergency hospitalisation (\%) }\end{array}$ \\
\hline $0-39$ & 38 & 57 \\
$40-79$ & 33 & 53 \\
$80-119$ & 45 & 66 \\
$120-159$ & 43 & 62 \\
$>160$ & $64^{\star \star}$ & $72^{\star}$ \\
\hline \multirow{2}{*}{ Diuretic adherence (\%) } & Patients undergoing a cardiac & Patients undergoing any \\
\hline$<80$ & 46 & hospitalisation (\%) \\
$80-124$ & 35 & 62 \\
$>125$ & 47 & 55 \\
& & 71 \\
\hline
\end{tabular}

${ }^{\star} \mathrm{p}<0.05$, test for trend; ${ }^{\star \star} \mathrm{p}=0.001$, Cochran-Armitage.

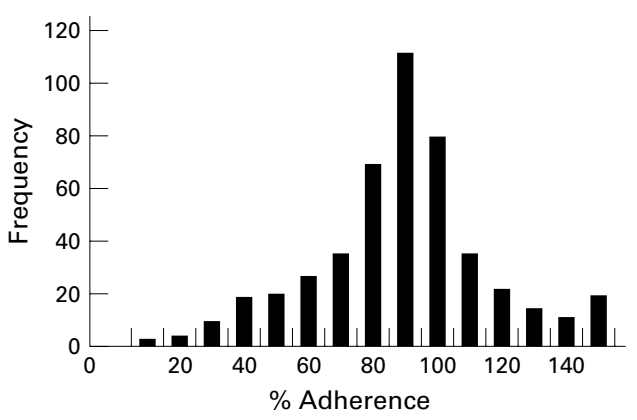

Figure 1 Adherence to diuretic treatment in $478 \mathrm{CHF}$ patients who were dispensed diuretic drugs.

hospitalisations of going from $39 \mathrm{mg} /$ day to $>160 \mathrm{mg} /$ day (38\% to $64 \%$ - a $68 \%$ increase) is only marginally greater than that of going from deprivation category $1-2$ to $5-6$ (26\% to $40 \%$ - a $54 \%$ increase). Nevertheless, diuretic adherence did not appear to influence hospitalisations for any cause.

Figure 1 shows the wide range of values for patient adherence with their diuretic regimen. In fact, $24 \%$ of patients appeared to ingest $<80 \%$ of their prescribed diuretic regimen. A reasonable proportion of patients appeared to be "over adherent", presumably because they replaced their tablets because they lost the previous supply. Neither age nor the absolute dose of diuretics prescribed influenced adherence with diuretic treatment, although the male patients had lower adherence rates (table 5).

\section{Discussion}

Our main finding is that social deprivation is independently associated with an increase in cardiac hospitalisations in those individuals who have previously had a myocardial infarc- 
Table 5 Association between age, sex, and diuretic dose on diuretic adherence during the study period

\begin{tabular}{ll}
\hline & $\begin{array}{l}\text { Mean diuretic adherence } \\
(\%)\end{array}$ \\
\hline Age (years) & \\
$46-55$ & 95 \\
$56-65$ & 86 \\
$66-75$ & 94 \\
$76-85$ & 92 \\
$85+$ & 92 \\
Sex & $91^{\star}$ \\
Men & 99 \\
Women & 98 \\
Diuretic dose (frusemide equivalents $\mathrm{mg} /$ day) \\
$0-39$ & 95 \\
$40-79$ & 94 \\
$80-119$ & 97 \\
$120-159$ & 94 \\
$>160$ &
\end{tabular}

tion and CHF. Importantly, this effect was independent of disease severity, as judged by the average diuretic dose, the death rate, and the duration of each hospital stay. It was also independent of non-adherence with diuretic treatment. Both the average prescribed dose of diuretic and the death rate are objective measures of chronic disease severity. A third measure of disease severity also included is the duration of hospital stay, which is a crude measure of the severity of each acute individual decompensation. Therefore, three different measures of disease severity could not account for the link between social deprivation and cardiac hospitalisations.

We found that social deprivation was mainly associated with the admission of a higher proportion of patients, which suggests that the threshold for hospitalisations in CHF may be lower in those with social deprivation. Previous data suggest the same overall phenomenon in that social deprivation increases hospitalisations in children, even though their use of general practitioner (GP) services or outpatient services is unaltered. ${ }^{2}$ Possible explanations for our findings could have been comorbidity, living alone, or poor social support at home. However, these seem unlikely, although not impossible, since the duration of each hospital stay was not influenced by social deprivation, whereas one would expect that comorbid conditions, living alone, and poor social support would prolong the duration of hospital stay.

Instead, the explanation probably lies in either patient behaviour, doctor behaviour, or a combination of both. With regard to patient behaviour, it could be that greater demand per unit of disease severity as a result of social deprivation reduces one's ability to cope with stress. ${ }^{8}$ Alternatively, it could simply be that the socially deprived patient accesses medical care rather differently - that is, they may be more likely to attend the hospital emergency department than call their GP. ${ }^{9}$ It is unlikely that the socially deprived patient calls the doctor at a later stage in each $\mathrm{CHF}$ decompensation since there was no effect of social deprivation on the length of each hospital stay. Doctor behaviour is another possible explanation. For example, a GP may be more liable to hospitalise a socially deprived patient because the doctor perceives that the socially deprived patient has poorer understanding of how to manage and overcome a clinical deterioration without being admitted to hospital. Another possibility is that the extra disease burden in socially deprived areas leads GPs to either use less preventive measures (such as flu vaccination) or to lower their threshold for hospitalisation because they have less time for repeated home visits to problem patients.

Adherence to medication is notoriously difficult to measure. We assessed adherence by whether the patient had enough tablets in the house to cover the appropriate time period. ${ }^{10}$ In this way, we are really measuring each patient's maximum possible level of adherence - that is, if the patient only had tablets available to cover $70 \%$ of the time, then this maximum possible level of adherence is $70 \%$, but it could be less if they did not swallow all available tablets. For the same reason, our figure of $24 \%$ of patients ingesting $<80 \%$ of their medication really means that non-adherence could be $>24 \%$ but it is very unlikely for it to be $<24 \%$. In our study, poor adherence was associated with being male rather than female but not with age, social deprivation, or diuretic dose. Since these data deal specifically with diuretic treatment, it is possible that diuretics caused more troublesome urinary symptoms in men than in women because of prostatism, leading to poorer adherence.

Diuretic adherence did not appear to influence hospitalisation. However, cross sectional data such as these are far from ideal as a means to examine this question since one might intuitively feel that a hospitalisation is likely to make a patient subsequently more adherent with their medication than they were before that admission, at least for a temporary period. Cross sectional data on adherence such as here would only produce a positive result if non-adherence was a very strong factor leading to hospitalisation. Our data can only be interpreted as saying that diuretic non-adherence is not an overwhelming reason for hospitalisation, but it could still be an important contributor. Our results leave open the possibility that non-adherence to diet, fluid restriction, or salt intake might still contribute. ${ }^{11}$

In conclusion, social deprivation was associated with an increase in cardiac hospitalisations in CHF patients. This effect was independent of disease severity and of non-adherence with treatment. Understanding how social deprivation influences doctor and patient behaviour in the important prehospital phase is now crucial in order to reduce the amplifying effect that social deprivation has on cardiac hospitalisations. Our data also open up the possibility that increased surveillance of socially deprived CHF patients by GPs, nurse practitioners or outpatient departments might reduce their currently high rate of cardiac hospitalisations. ${ }^{11}$

We thank all the doctors in Tayside over many years who contributed to the SMR1 database.

1 Blaxter M. Equity and consultation rates in general practice. BMF 1984;288:1963-7.

2 Cooper H, Smaje C, Arber S. Use of health services by children and young people according to ethnicity and social class: secondary analysis of a national survey. BMF 1998;317:1047-51. 
3 Collins E, Klein R. Equity and the NHS: self reported morbidity, access and primary care. BMF 1980;28:1111-15. Duffield JS, Craig K, Plant WD. Patterns in acute referral to hospital. Scott Med f 1997;42:105-7.

5 McMurray J, Hart W, Rhodes G. An evaluation of the cost of heart failure to the NHS in the UK. Br f Med Economics 1993;6:99-110

6 Evans JMM, McDevitt DG, MacDonald TM. The Tayside medicines monitoring unit (MEMO): a record-linkage system for pharmaco-vigilance. Pharmaceutical Medicine 1995; 9:177-84.

7 McLoone P. Carstairs codes for Scottish postcode sectors from the 1991 census. Glasgow: Public Health Research Unit, University of Glasgow, 1991.
8 Lewis G, Bebbington P, Brugha T, et al. Socio-economic status, standard of living and neurotic disorder. Lancet 1998;352:605-9.

9 Watson JP, Cowen P, Lewis RA. The relationship between asthma admission rates, routes of admission and socioeconomic deprivation. Eur Respir f 1996;9:2087-93.

10 Morris AD, Boyle DIR, McMahon AD, et al for DARTS/ MEMO collaboration. Adherence to insulin treatment, glycaemia control and ketoacidosis in insulin dependent diabetes mellitus. Lancet 1997;350:1505-10.

11 McMurray J, Stewart S. Nurse led multidisciplinary intervention in chronic heart failure. Heart 1998;80:4301.

\section{IMAGES IN CARDIOLOGY}

\section{Traumatic aortopulmonary fistula: echocardiographic appearance}

A 36 year old man was admitted to a district general hospital, following a stab wound to his chest, in a state of circulatory collapse. He was resuscitated during emergency surgery with control of profuse bleeding from the front of his pulmonary artery.

He was transferred to the regional cardiothoracic unit for postoperative care. The following day a continuous murmur was heard at the second left intercostal space. Transoesophageal echocardiography (left) demonstrated a traumatic aortopulmonary fistula (APF) just above the aortic valve close to the left main coronary

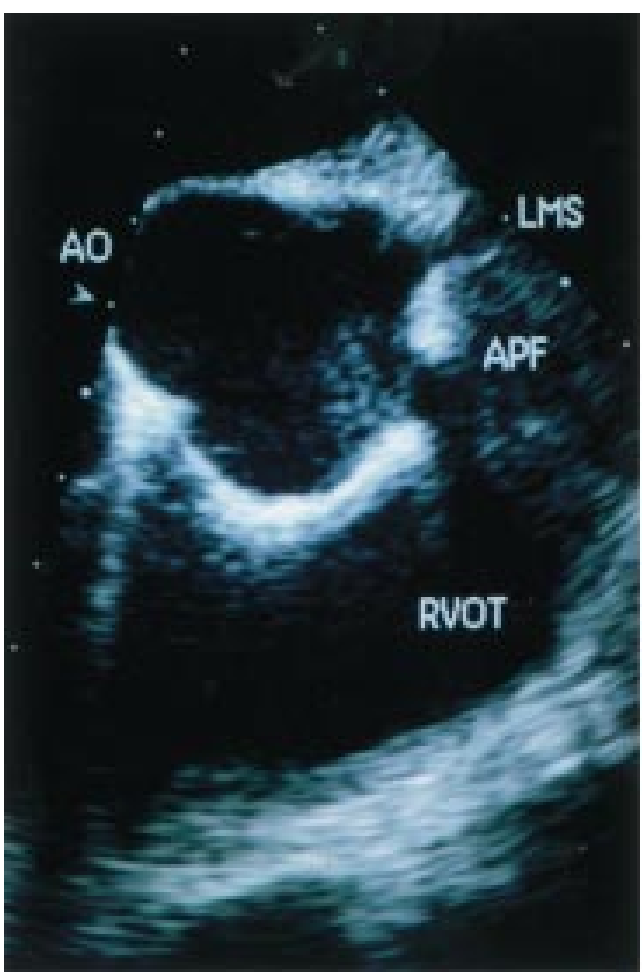

artery (LMS). Colour Doppler (right) showed flow across the fistula (AO, aorta; RVOT, right ventricular outflow tract). He developed fulminant pulmonary oedema and underwent urgent reoperation 48 hours after the first operation. During cardiopulmonary bypass, the fistula was closed by simple suture to lacerations of the aorta and posterior main pulmonary artery. Injury to the pulmonary valve leaflets was not repaired but the entry wound in the pulmonary valve sinus was resutured. He made a good postoperative recovery and was discharged home one week later.

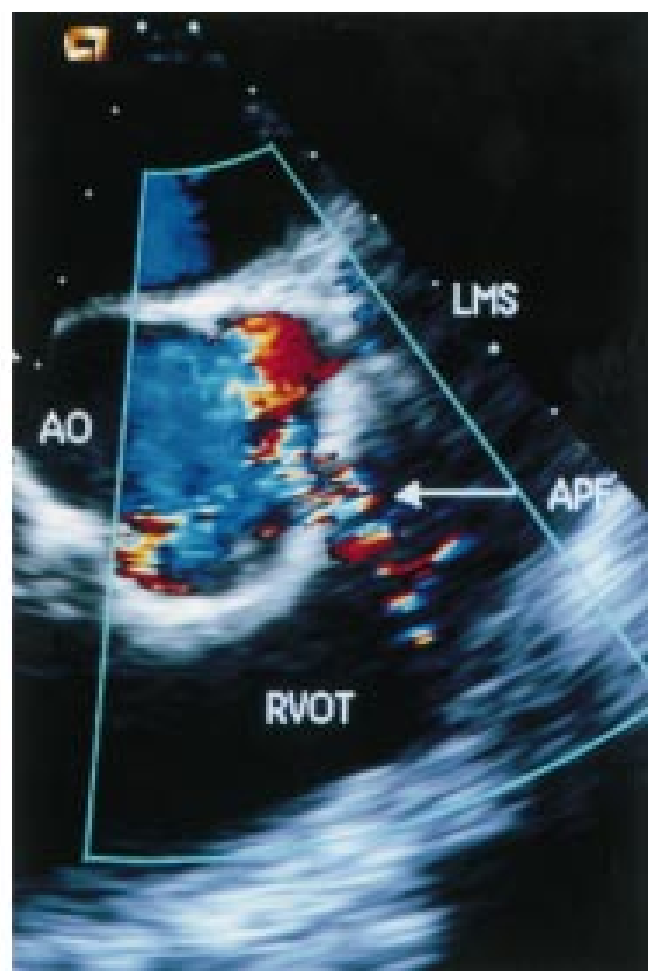

Dr Vincent Moohan is acknowledged for providing the transoesophageal echocardiogram.

J MARK JONES

DENNIS J GLADSTONE CHRISTINE MAGUIRE 